\title{
Unified surface and volumetric projection of physiological imaging data
}

\author{
Thomas F. Kirk ${ }^{a, b, *}$, Martin S. Craig ${ }^{c, d}$, Michael A. Chappell ${ }^{c, d, b}$ \\ ${ }^{a}$ Institute of Biomedical Engineering, Department of Engineering Science, University of \\ Oxford \\ ${ }^{b}$ Wellcome Centre for Integrative Neuroimaging, Nuffield Department of Clinical \\ Neurosciences, University of Oxford \\ ${ }^{c}$ Mental Health and Clinical Neurosciences, School of Medicine, University of Nottingham \\ ${ }^{d}$ Sir Peter Mansfield Imaging Centre, School of Medicine, University of Nottingham
}

\begin{abstract}
Projection of volumetric data onto the cortical surface is an important precursor to performing surface-based analysis. Numerous projection methods have been reported in the literature, many of which make assumptions which tie them to use with specific modalities, notably blood oxygenation level dependent (BOLD) imaging. This means that they may not be appropriate for use with modalities where subcortical tissue contributes a signal of interest. This work details a new projection that provides a number of generalisations and extensions to existing methods. Namely, it may be used to project arbitrary data without making modality-specific assumptions and can produce unified surface and volumetric representations of data (a concept also known as grayordinates space). When constrained to the same assumptions as existing methods, a comparison using simulation data shows that it produces similar outputs. When these assumptions are relaxed to project simulation data containing both cortical and subcortical signals to and from a unified surface and volume space, substantial and statistically significant differences in recovery of ground truth are observed compared to existing methods.
\end{abstract}

Keywords: projection, surface, volumetric, partial volume, hybrid

\footnotetext{
*Corresponding author. Postal address: c/o Prof. M. Chappell, School of Medicine, Queen's Medical Centre, Nottingham, NG7 2UH, UK

Email address: tomfrankkirk@gmail.com (Thomas F. Kirk)
} 


\section{Introduction}

The cerebral cortex has a thin and highly folded structure that may be thought of as a two-dimensional surface. Many properties of the cortex, notably the organisation of cortical areas, are best understood in the context of this surface topology and geometry [1]. In particular, on the assumption that the function of cortical areas will correlate closer according to geodesic (along the surface) than geometric (straight line) distance [2], surface-based analysis strategies should be inherently better suited to the study of the cortex than volumetric strategies, which divide the region of interest into voxels with no regard to underlying anatomy. Reported benefits of surface-based analysis of physiological imaging data include reduced bias and variance in kinetic modelling of positron emission tomography (PET) data [3; improved localisation of functional areas within the cortex for blood oxygen level dependent (BOLD) magnetic resonance imaging (MRI) data [4, 5]; and improved inter-subject registration for multi-modal imaging [5, 6].

All surface-based analysis of volumetric data must start with a means of forward projecting data from the volume 'onto the surface'. This is a complex operation for which there is no single analytic solution; instead, the methods that exist encode various assumptions about anatomy and the signal generation mechanism for the modality in question. Particularly important is the existence of the partial volume effect (PVE), a consequence of the low spatial resolution of imaging voxels in relation to the thickness of the cortex, which results in multiple tissues being present in a given voxel 7,8 . PVE in voxels intersecting the cortex means that the acquired signal is likely to be a mixture of signal from cortical grey matter (GM), subcortical white matter (WM) and cerebrospinal fluid (CSF). Hence, a desirable feature of any projection method is that it is partial volume aware and attempts to minimise the amount of non-cortical signal that is projected onto the surface.

The majority of existing projection methods have been developed for use 
with BOLD data, which has important implications for how they treat WM signal. For example, Grova et al. expressly ignored WM signal in their method [9, whereas Operto et al. started by deriving the expected distribution of the BOLD signal on either side of the cortical ribbon, including in subcortical WM [10]. Similarly, the method developed by Glasser et al. is intended to be followed

35 by a regression to remove any WM signal that has been projected to the surface [11. These characteristics mean that these methods may not be suitable for the projection of data that does contain a WM signal of interest, and/or a WM signal that is spatially varying, for example that produced by arterial spin labelling (ASL).

Due to the fact that a small number of voxels in a typical volumetric dataset intersect the cortex, a surface-based analysis strategy will by design ignore the vast majority of imaging voxels. This is of no consequence if the cortex is the sole anatomy of interest, but if this is not the case, then a separate analysis must be run on subcortical voxels (likely a volumetric analysis, because the subcortex 45 is not surface-like). Such a dual surface and volumetric analysis strategy has, for example, been adopted by the Human Connectome Project (HCP) [12, 11]: from a single timeseries of BOLD data, a conventional volumetric analysis is performed for the subcortex, whilst a surface analysis is performed for the cortex on a surface-projected copy of the data, maximising the extraction of useful ${ }_{50}$ information. The results of these separate analyses are then unified in grayordinates space, a domain of representation that allows data to be recorded both on the surface for the cortex and in the volume for the subcortex 1 A notable aspect of this strategy is the bifurcation that is required: whilst the point of departure is a single volumetric timeseries, and the end-point is a single set of analysis results in grayordinates space, two parallel processing pipelines are required in between.

The objective of this work was to build a projection framework, which has

\footnotetext{
${ }^{1}$ The CIFTI file format embodies this dual representation explicitly [13].
} 
been incorporated into the exiting Toblerone software packag $2^{2}$, that extends existing methods in the following manner. Firstly, by providing the ability to perform forward (volume to surface) and reverse (surface to volume) projection, something that is not true of all existing methods. Secondly, by permitting the projection of arbitrary data, without making modality-specific assumptions, in particular data containing WM signal. Finally, by expanding the scope of projection to include unified surface and volumetric data representations, i.e., projection to and from a grayordinates-like space.

\section{Theory}

\subsection{Hybrid space}

Hybrid space is a generalisation of the HCP's concept of grayordinates. It is a domain of data representation formed by taking the union of the surface and volume spaces, such that data in hybrid space has both a vertex-wise (surface) and voxel-wise (volume) representation for the cortex and subcortex respectively. For any space, be it surface, volumetric or hybrid, the spatial locations at which data values are recorded are hereafter referred to as nodes. Thus, in hybrid space, the set of nodes on which signal values are recorded comprises all surface vertices and all voxels of interest, as illustrated in figure 1 .

An important property of hybrid space is that it provides an explicit representation of PVE. This follows from the fact that nodes in hybrid space are defined to only ever map to a single tissue type (which may be GM or WM, depending on where the node itself is). Voxels that are affected by PVE will therefore contain multiple nodes (for example, a subcortical WM node and some cortical GM nodes), as is the case for many of the voxels in figure 1 . Toblerone can perform projection between the volume and surface spaces (a conventional mode of operation that is directly comparable with existing methods), or it can

\footnotetext{
${ }^{2}$ https://github.com/tomfrankkirk/toblerone
} 


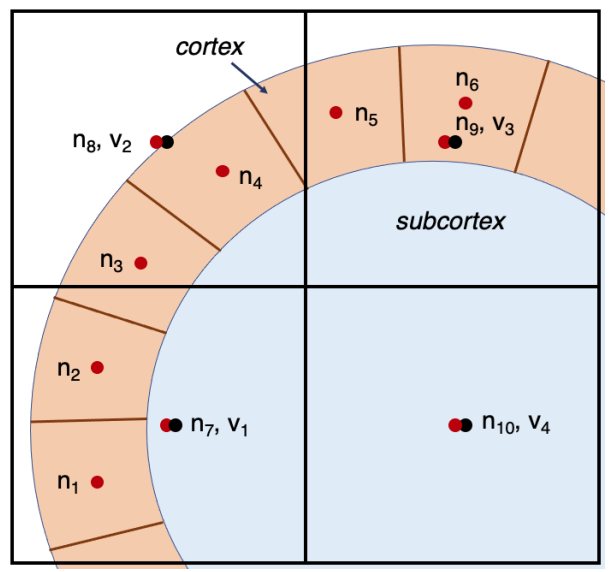

Figure 1: Schematic representation of voxels and nodes in hybrid space. The primitives comprising the cortical ribbon are denoted with brown boundaries. Voxel boundaries and centres are in black, labelled $v_{1}$ to $v_{4}$. Nodes are in red; $n_{1}$ to $n_{6}$ are cortical (corresponding to surface vertices), $n_{7}$ to $n_{10}$ are subcortical (corresponding to voxels). Individual voxels may contain multiple nodes, which is an explicit representation of PVE.

perform projection between the volume and hybrid spaces (a new capability for which there are no existing methods).

As nodes in hybrid space correspond to only one tissue, the signals associated with them must also respect this distinction. Signal on cortical nodes is considered as being purely cortical (i.e., GM) in origin, and signal on subcortical nodes is considered as purely subcortical in origin (i.e., WM or a subcortical GM structure). How these separate signals are derived from data that contains PVE is beyond the scope of this work (and is in fact the objective of partial volume effect correction, $\mathrm{PVE}^{3}$ ). For the purposes of this work, it is only necessary to posit that the separate signals exist and that hybrid representation respects their separation. It follows that, when projecting from hybrid to volume space, the process of PVE must be recreated to mix the separate tissue signals within

\footnotetext{
${ }^{3}$ Though many projection methods acknowledge the existence of PVE within data, there is little that can be done to remove this during the narrow operation of projection. To do so would require a basis on which to separate cortical and subcortical signals, which is a modality-specific consideration and explains why PVEc is a non-trivial operation.
} 
each voxel in the correct proportions.

\subsection{Construction of the projection}

The projection is constructed from mesh representations of the inner and outer surfaces of a cortical hemisphere, produced for example by FreeSurfer (in which case they are referred to as the white and pial surfaces respectively) [14. Mesh representations consist of vertices (points in 3D space) and triangles (triplets of vertex numbers denoting how vertices should be connected to form the discrete primitives of the surface). Triangle correspondence between the inner and outer surfaces of the cortex is assumed. Though meshes of any resolution can be used, it is envisaged that meshes at $32 \mathrm{k}$ resolution are sufficient for physiological data (as is the case for HCP analysis pipelines [11]). Though the inner and outer surfaces of the cortex are used to define the extents of the cortical ribbon, the actual projection itself is made onto the cortical midsurface by default, though this could be modified for other use cases such as laminar fMRI. A set of PV estimates for the cortex is also used; these are produced using Toblerone's previously-reported PV estimation function operating on the cortical surfaces [15]. Both the forward and reverse projections are constructed from the two matrices that are introduced in the following paragraphs.

Voxel-triangle matrix. The voxel-triangle matrix performs a mapping between individual voxels and the volume primitives that comprise the cortex. Construction of the primitives is approached in a triangle-wise manner, as opposed to the vertex-wise approach adopted by other methods, as this allows for the construction of extremely simple primitives. For each pair of triangles on the inner and outer surfaces, their corresponding vertices are connected to form a triangular prism 4 which is subdivided into three non-overlapping tetrahedra [16. For example, for triangles with vertices $\triangle A B C$ and $\triangle a b c$ on the outer and inner surfaces respectively, the prism may be split into tetrahedra $A B C a, C a b c$

\footnotetext{
${ }^{4}$ These are similar to a Toblerone bar, hence the name.
} 
and $B C a b$. As a consequence of this subdivision, each quadrilateral face of the prism is split along the diagonal into two triangles. It is important to ensure that the orientation of this face diagonal is consistent between neighbouring prisms so as to avoid producing overlapping tetrahedra. This is achieved by fixing the direction of subdivision according to the ordering of vertex numbers: 'positive' if $A<B, B<C$ or $C<A$, and 'negative' otherwise.

Subsequently, for each voxel in the neighbourhood of the prism, an isotropic grid of sample points is initialised. Figure 2 shows one such prism and the sample points from one neighbourhood voxel. Sample points are tested to determine if they lie within each tetrahedra (via the Delaunay triangulation [17]) and the results stored in the matrix VT. The elements $\mathrm{VT}_{v, t}$ are continuous values in the range $[0,1]$ recording the fraction of sample points from voxel $v$ that 135 lie inside triangular prism $t$. This matrix is highly sparse: for a voxel size of $2.2 \mathrm{~mm}$ isotropic and a surface resolution of $32 \mathrm{k}$ vertices, each triangular prism intersects around 10 voxels on average.

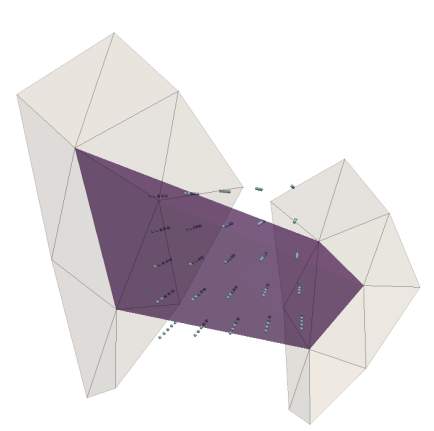

(a)

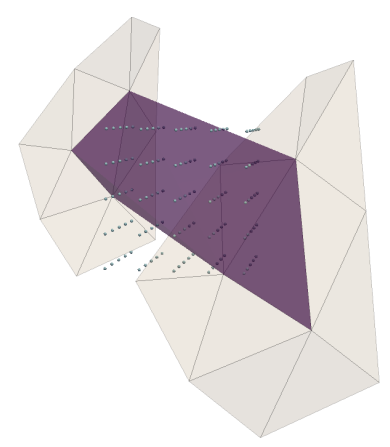

(b)

Figure 2: Two alternative views of the triangular prism (purple) formed by connecting one pair of corresponding triangles on the inner and outer surfaces (grey) of the cortex. The subdivision into three tetrahedra is not shown. A $5^{3}$ grid of sample points are tested to determine the volume of intersection between the voxel and prism, which is recorded in the voxel-triangle matrix. 
Points-triangle matrix. The points-triangle matrix records the area of each triangle on the cortical midsurface that is associated to its individual vertices (though vertices are now referred to as points to avoid re-using the letter $v$ ). These values are calculated using the Voronoi region approach, extended to arbitrary meshes by Meyer et al. [18, and are recorded in the matrix PT. The element $\mathrm{PT}_{p, t}$ denotes the area of triangle $t$ that is associated to point $p$. Once again, this matrix is highly sparse as each point is contained by only a few triangles.

Forward projection: volume to surface. To form the volume to surface projection, the VT matrix and PT matrices are first normalised in a row or columnwise sense, denoted with the $\|\cdot\|_{\text {row }}$ operator for the row-sense. This operator rescales rows or columns with any non-zero elements such that the row or column sum is unity. For the VT matrix, this is equivalent to ensuring that the sum of voxel weights for each triangle is unity, and for the PT matrix, that the sum of triangle areas for each point is unity. The effect of this is to turn these individual matrices into weighted-averaging operators that preserve signal intensity. The forward projection is then defined by the multiplication

$$
\mathrm{VS}=\|\mathrm{PT}\|_{\text {row }}\|\mathrm{VT}\|_{\text {col }}^{T}
$$

Forward projection: volume to hybrid. In order to extend the forward projection into hybrid space, it is necessary to include the mapping between voxels and subcortical nodes (the VS matrix accounts only for cortical nodes). This is achieved by concatenating the VS matrix with the identity $\mathrm{I}_{v}$ matrix of size (number of voxels) to yield VH. This implies a one-to-one mapping for all voxels to their hybrid space counterpart (it is assumed, but not required, that irrelevant voxels are masked out, in which case their corresponding nodes are also removed).

$$
\mathrm{VH}=\left[\frac{\mathrm{VS}}{\mathrm{I}_{v}}\right]
$$


Forward projection: edge up-scaling. Depending on the nature of the data that is to be projected, edge up-scaling is an optional extra step that can be incorporated into the forward projection. For signals that scale directly with the amount of tissue in a voxel, signal magnitude will be artificially low in any edge voxels that are less than $100 \%$ brain tissue (for example, those that contain some CSF which does not contribute any signal). In this scenario, it may be advantageous to up-scale the signal to account for the lost intensity. This is performed by calculating the voxel-wise brain PV (the sum of GM and WM PVs), taking the inverse of this quantity to form an upweight vector $\mathbf{u}$, and finally taking the outer product (denoted $\otimes$ ) with the constituent parts of the volume to hybrid projection matrix.

$$
\mathrm{VH}_{\text {edge }}=\left[\frac{\mathbf{u} \otimes \mathrm{VS}}{\mathbf{u} \otimes \mathrm{I}_{v}}\right]
$$

The purpose of this step is to perform a very simple correction for signal lost due to PVE, with the important caveat that it will be poorly-conditioned in voxels that contain a low GM and WM PV. Hence, this correction may not be appropriate for noisy data, but may be useful for data that has already undergone some form of processing. An example of where this step could be applied is projection of ASL data (the magnetisation signal scales with tissue PV); an example of where it would not be appropriate is projection of a timing volume (such as voxel-wise MRI slice times, which are independent of tissue $\mathrm{PV})$.

Reverse projection: surface to volume. To form the surface to volume projection, the VT and PT matrices are normalised in the opposite sense to before. For VT, this ensures that the sum of triangle weights for each voxel is unity, and for PT, that the sum of vertex areas for each triangle is unity. The surface to volume projection is then defined by the following multiplication.

$$
\mathrm{SV}=\|\mathrm{VT}\|_{\text {row }}\|\mathrm{PT}\|_{\text {col }}^{T}
$$


Reverse projection: hybrid to volume. In order to form the reverse projection from hybrid space, PVE must be recreated. This follows from the fact that nodes in hybrid space enforce the separation of signals from different tissues. Each row of the hybrid to volume projection matrix corresponds to a voxel, and the column values record the weights in which both cortical and subcortical nodes contribute to that voxel. A set of voxel-wise GM and WM PV estimates are then used to scale the overall contribution of cortical and subcortical nodes within each voxel, where $\mathbf{b}=\mathbf{p} \mathbf{v}_{g m}+\mathbf{p} \mathbf{v}_{w m}$ represents the voxelwise sum of $\mathrm{PV} \$$ In particular, each row of the surface-to-volume matrix is scaled by the GM PV of the corresponding voxel, and each row of the identity by the WM $\mathrm{PV}$ of the corresponding voxel.

$$
\mathrm{HV}=\left[\frac{\mathbf{p} \mathbf{v}_{g m}}{\mathbf{b}} \otimes \mathrm{SV} \mid \frac{\mathbf{p} \mathbf{v}_{w m}}{\mathbf{b}} \otimes \mathrm{I}_{v}\right]
$$

Reverse projection: edge down-scaling. Edge down-scaling may optionally be applied for the reverse projection depending on the nature of the data in question. The same principles as for the forward case apply: if the signal is expected to scale with tissue $\mathrm{PV}$, then edge scaling reduces the projected signal in voxels that are less than $100 \%$ brain tissue. In this situation, there is a subtle distinction between PVE caused by mixed signal and PVE caused by missing signal, though both originate in the same manner (the presence, or absence, of multiple tissues in a voxel). Edge down-scaling is achieved by assembling the constituent parts of the hybrid to volume projection matrix without the weighted averaging implied by the brain PV $\mathbf{b}$.

$$
\mathrm{HV}=\left[\mathbf{p v}_{g m} \otimes \mathrm{SV} \mid \mathbf{p v}_{w m} \otimes \mathrm{I}_{v}\right]
$$

\footnotetext{
${ }^{5}$ Note the double-scaling here, which reflects the slightly different types PVE at work. Firstly, there is the question of determining the relative contributions of the different cortical nodes within the voxel (a weighting between neighbouring GM nodes); secondly, there is the question of determining the relative contributions of the cortical versus subcortical signals (the overall ratio of GM to WM).
} 
Though existing methods have largely focussed on the forward projection

[20]. For experiments involving a round-trip of projection (introduced in section 3.2.1, the lsQR variants of each method were run at the intermediate step as a 
means of completing the round-trip. For example, when using Toblerone to perform a surface (A) to volume (B) to surface (C) projection, the Toblerone-lsQR variant was constructed by using lsQR on the intermediate data $\mathrm{B}$ in order to reach C. It is important to note that lsQR does not yield an inverse projection in the general sense; rather, for a specific vector of projected data, it is able to invert the projection for that data only. This is contrast to Toblerone and RC that define all of their projections independently of the data to be projected.

Comparisons were performed in both the forward (volume to surface) and reverse (surface to volume) directions. Where possible, differences between the methods were tested for statistical significance using a paired $t$-test of independent measures where equal variances were not assumed and the significance threshold was set at $p=0.01$.

\subsection{Datasets}

Two types of data were simulated, with local and global signal distributions respectively. For both data, the underlying anatomical information was taken from the structural pre-processed output of the 45 subject test-retest cohort of the Human Connectome Project, onto which physiological data were simulated [11. Left white and pial cortical surfaces at $32 \mathrm{k}$ mesh resolution were used to define cortical anatomy. Whilst Toblerone is able to perform simultaneous projection to the left and right cortical hemispheres in a single operation, the $\mathrm{RC}$ method is not, and hence the comparison was restricted to consider single hemispheres only.

Local cortical signal. The first dataset, defined for the surface space only, models signals that are local in nature. The principle, previously used by both Grova and Operto, was to simulate small areas of activation within the cortex by selecting a random vertex on the cortical midsurface (with local thickness greater than $2 \mathrm{~mm}$ to avoid areas with high partial voluming), and applying 265 the following signal values to the point, its first-order neighbours, second-order, and so on: $[1,1,0.9,0.7,0.5,0.3,0.1]$. All other vertex values were left at zero. 
Unlike the work of Grova and Operto, the location of the activation was allowed to vary between subjects; one such activation is shown in figure 3 . Toblerone (in surface/volume mode) and RC were used on this dataset.

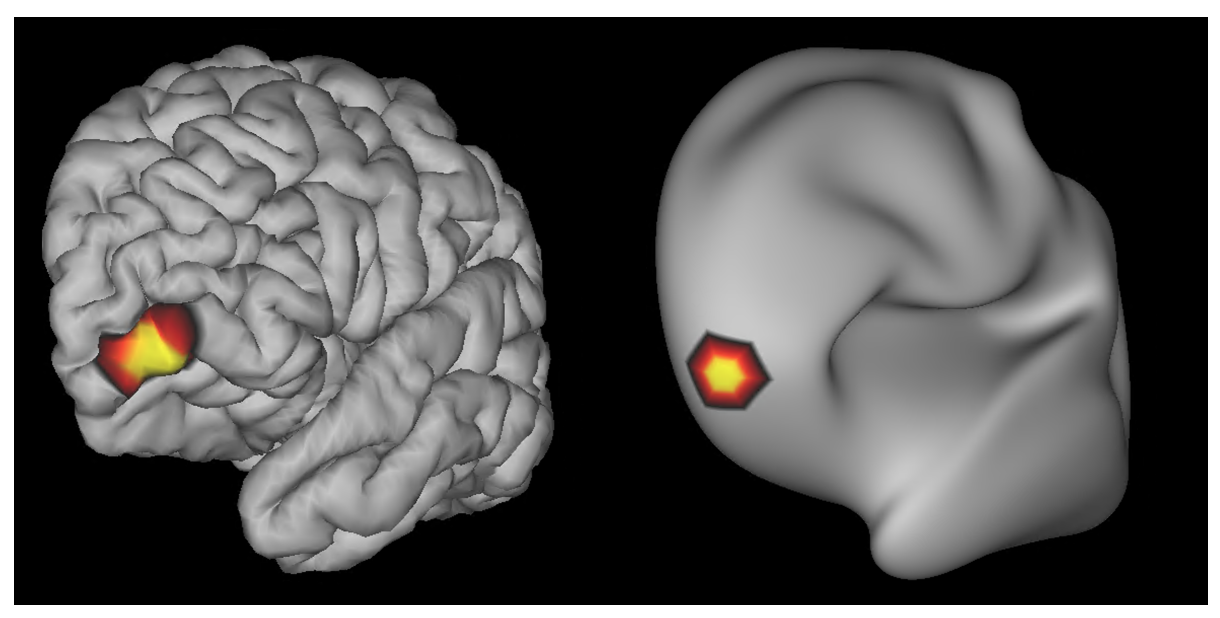

Figure 3: Example activation for subject 103818 of the HCP. The same signal map is shown on both the pial surface (left) and inflated surface (right).

Global cortical signal. The second dataset, defined for both the surface and volume spaces, models a global signal in the cortex only. Two different variants were tested: one with a uniform signal of 60 a.u., and one with sinusoidal variation of $60 \pm 18$ a.u. The volumetric data were produced by evaluating volumetric 3D fields of values and then performing a voxel-wise multiplication with subject-specific cortical GM PV estimates obtained via Toblerone's surfacebased PV estimation. In order to produce surface data, trilinear interpolation of the same volumetric fields onto each subject's midsurface was performed. This process is illustrated in figure 4 Toblerone (in surface/volume mode), RC, Toblerone-lsQR and RC-lsQR were used on this dataset. 


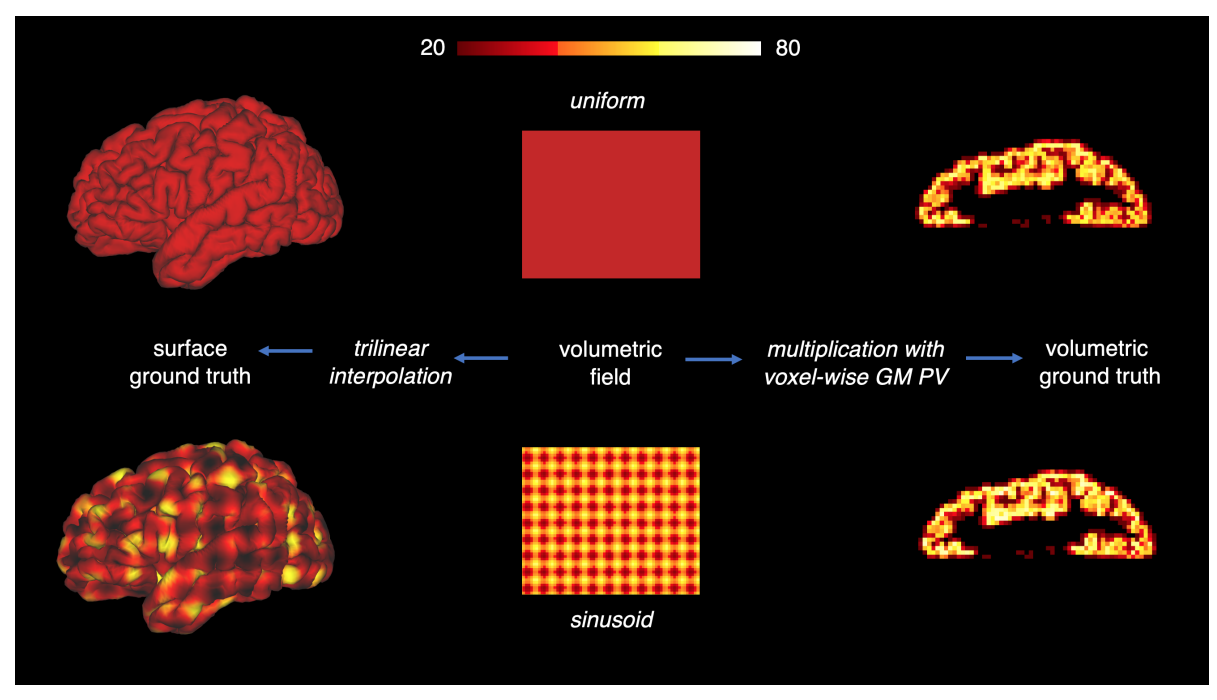

Figure 4: Generation of ground truth surface and volume data for both uniform and sinusoid signals.

Global cortical and subcortical signal. The final dataset, defined for both the surface and volume spaces, models a global cortical and subcortical signals. The cortical signal was generated in the same manner as for the global cortical signal dataset, onto which the following subcortical signals were added. For the uniform dataset, a constant value of 20 in all of the subcortex, and for the sinusoidal variation, a field of $20 \pm 2$. In the volume space, the cortical and subcortical signals were combined in proportion to voxel-wise GM and WM PV estimates. In the hybrid space, the ground truth was generated by concatenating a vector of subcortical signal values to the vector of cortical values. For simplicity, the subcortex was taken to contain only WM and no deep subcortical GM structures; in any case, because the projections consider only voxels intersecting the cortex, this anatomical inaccuracy is of no consequence. Toblerone (in hybrid/volume mode), RC, Toblerone-lsQR and RC-lsQR were used on this dataset, though the RC methods deal only with surface nodes and are unaware of the presence of subcortical nodes, so the comparison is incomplete. 

data, with ASL it is typically desired to perform absolute quantification and hence normalisation is inappropriate. A parallel analysis of the same dataset was thus performed on round-trip projected data without normalisation and 

indicative of the PVE-induced scaling incorporated into each projection method. Secondly, the maximum signal value when projected back into the surface space. Though neither method will be able to perfectly reproduce the ground truth (due to PVE), the extent to which signal intensity is preserved 330 is nevertheless important for absolute quantification strategies ${ }^{6}$. Finally, the ratio of activated vertices to ground truth activated vertices after the roundtrip projection, as this indicates the extent to which the projection has smoothed the original signal distribution.

Global cortical (and subcortical) signal. These experiments were run on the volumetric ground truths were taken through a round-trip of projection, as illustrated in figure 5. The lsQR variants of each projection were also used at the intermediate step to obtain the inverse projection. The following metrics were calculated with respect to ground truth: root-mean-square (RMS) error, and minimum and maximum signal values, to investigate how signal intensity and range was preserved. The latter metrics are of interest because an ideal projection should not produce signal values that are outside of the input signal range. In the context of these experiments, where the ground truth data were always positive, this means projection should never yield a negative signal value, or value above $100 \%$ of the ground truth maximum.

\footnotetext{
${ }^{6}$ Though Toblerone does implement edge up-scaling, this was not included in the interests of a like-for-like comparison with RC.
} 


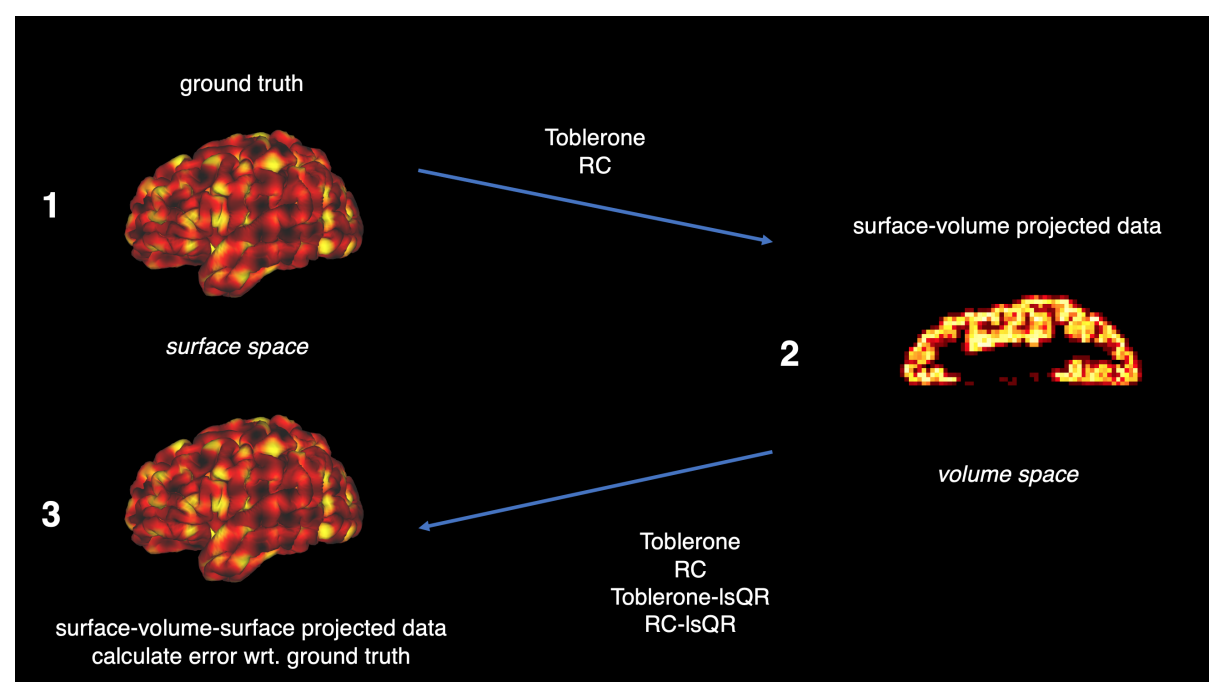

Figure 5: Schematic of the surface to volume to surface round-trip projection experiment. Both Toblerone and $\mathrm{RC}$ were used to project ground truth surface data into the volume. The volumetric data were then projected back to the surface using two methods: each method's self-inverse (i.e., Toblerone's volume to surface), or the lsQR inversion of each method's forward projection (i.e., Toblerone-lsQR in this case). Finally, metrics were calculated with respect to ground truth in the volume space. The volume to surface to volume experiment was of the same form, with the spaces swapped around.

\subsection{Ethics statement}

As this work is computational methods development using previously published public datasets, no further ethical review was performed besides that of

the original Human Connectome Project [12].

\section{Role of the funding source}

None of the funders of this work were involved in study design; data collection, analysis or interpretation; the preparation of this manuscript; or the decision to submit for publication. 


\section{Results}

\subsection{Local cortical signal}
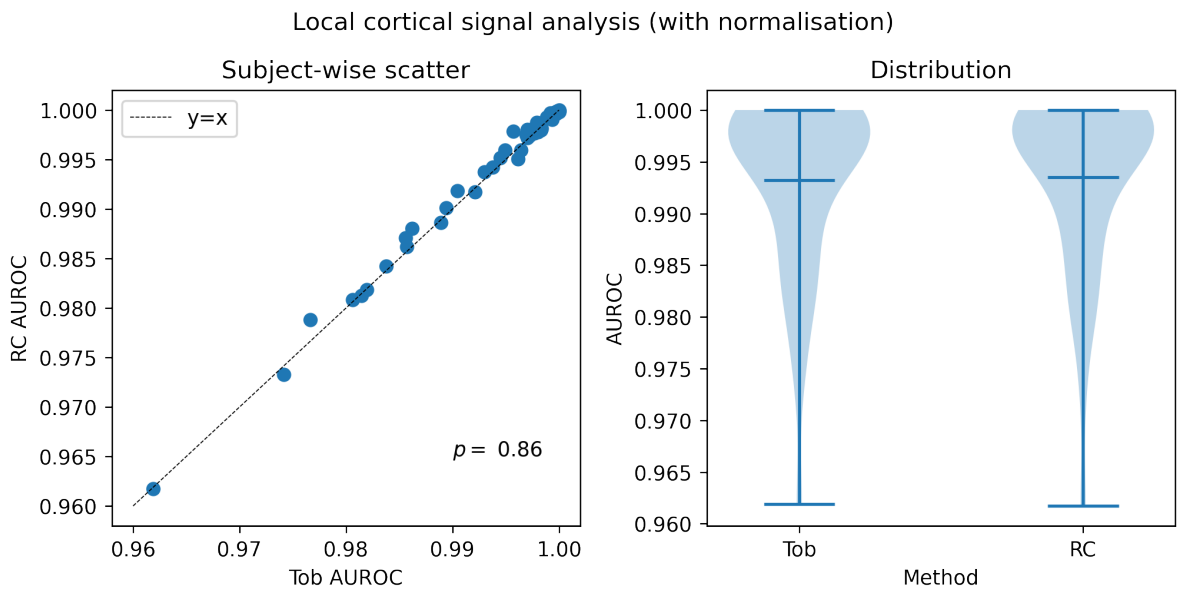

Figure 6: AUROC scores for the round-trip projected local cortical signal data. Right: a per-subject scatter plot of scores revealed no statistically significant difference between the two methods. Left: both methods returned extremely similar distribution of scores across subjects.

Figure 6 shows the AUROC analysis on round-trip projected local cortical signal surface data with normalisation. Both methods returned extremely similar outputs, to the point where no statistically significant difference could be detected. 

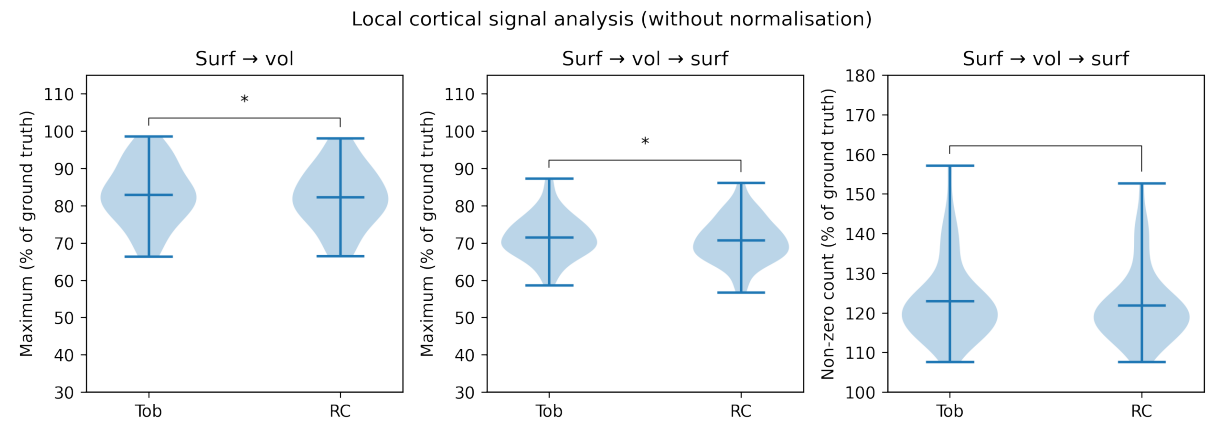

Figure 7: Round-trip projected local cortical signal surface data without normalisation. Left: distribution across subjects of maximum signal value after projection into volume space. Centre: distribution across subjects of maximum signal value after projection back onto the surface. Right: the distribution across subjects of non-zero vertices, as a proportion of ground truth, was slightly wider and of greater mean for Toblerone, though the difference was not statistically significant. * denotes a comparison of statistical significance.

Figure 7 shows the analysis of round-trip projected local cortical signal data without normalisation. Both methods returned extremely similar distributions of maximum signal values for projection into the volume or back onto the surface, though in both cases there was a small and statistically significant advantage in favour of Toblerone. When considering the number of non-zero vertices following round-trip projection, expressed as a fraction of the ground truth, Toblerone returned a distribution slightly wider and with higher mean than RC. 


\subsection{Global cortical signal}

Global cortical signal, volume $\rightarrow$ surface $\rightarrow$ volume
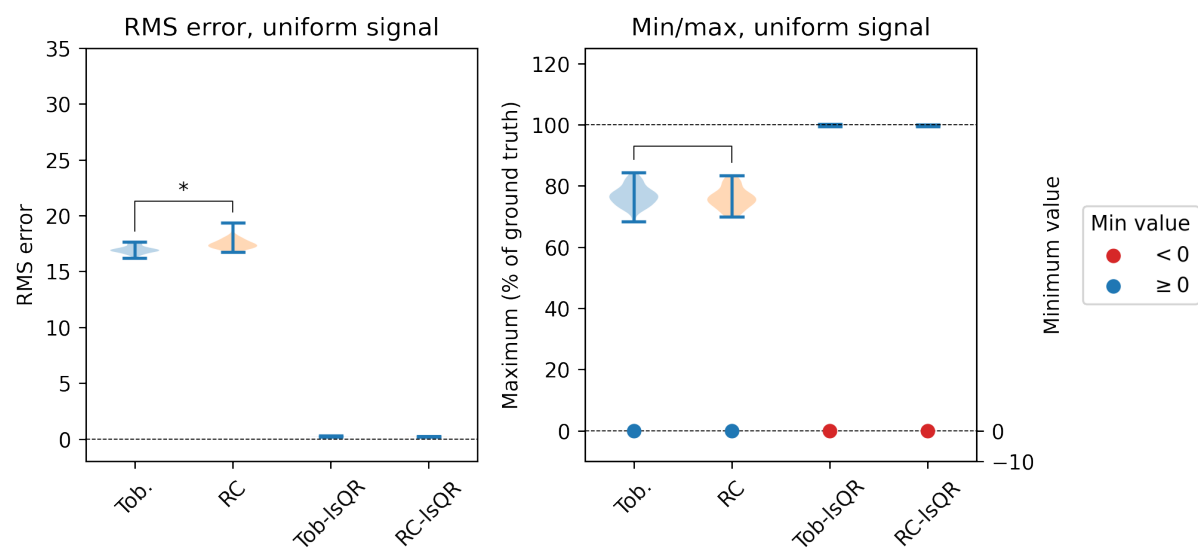

Figure 8: Global cortical signal, uniform value, round-trip projection from volume to surface to volume. Left: Toblerone produced significantly lower RMS errors than RC of around 17, whereas lsQR methods returned almost no error. Right: both Toblerone and RC returned similar maximum signal values of around $80 \%$, with no significant difference between the two, and both returned zero signal minimums. lsQR methods returned almost maximum signal values of almost $100 \%$ of ground truth, but the minimum signal values were slightly negative. * denotes a comparison of statistical significance.

Figure 8 shows the results of the round-trip projection on global cortical signal data, starting and ending in the volume space. In terms of RMS error, Toblerone performed better than RC (statistical significance was reached), whereas for maximum signal values both methods performed similarly. By contrast, lsQR methods returned essentially 'ideal' values for RMS error and maximum signal, though at the cost of slightly negative minimum signal values.

The complete results from this experiment (given in the supplementary material) were in agreement with the subset presented in figure 8 the RMS errors produced by Toblerone were always somewhat lower than RC (statistical significance was reached); and the maximum values produced by both methods were essentially identical (no significant difference observed). lsQR methods produced lower RMS errors than their corresponding original method (ie, ToblsQR lower than Toblerone itself); but minimum and maximum signal values for 
these methods were observed to fall outside the desired range of zero to $100 \%$ of ground truth.
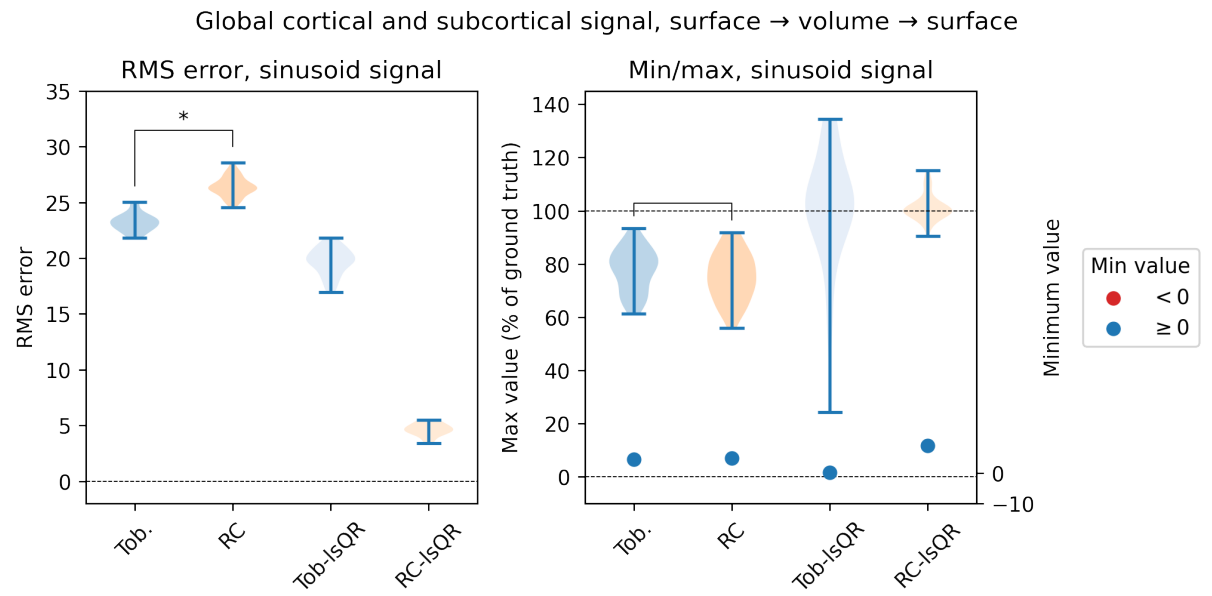

Figure 9: Global cortical and subcortical signal, sinusoid variation, round-trip projection from surface to volume to surface. Left: Toblerone returned significantly lower RMS errors than RC (around 24 versus 26 a.u.). RC-lsQR performed substantially better than ToblsQR, and both lsQR methods performed better than their original counterparts. Right: maximum and minimum signal values. Toblerone and RC returned similar maximums, with minimums somewhat above zero. lsQR methods, particularly Tob-lsQR, returned maximum signal values substantially above $100 \%$ of ground truth, up to $130 \%$ in the extreme case. * denotes a comparison of statistical significance.

Figure 9 shows the results of the round-trip projection on global cortical and subcortical sinusoid variation data, starting and ending in the surface space. Toblerone produced lower RMS errors than RC (statistical significance was reached); both lsQR methods returned lower errors but were still substantially greater than zero. Both Toblerone and RC returned maximum signal values around $80 \%$ of ground truth, whereas lsQR methods returned a wide range of values that were often greater than $100 \%$. All methods returned minimum values somewhat above zero. 
The complete results from this experiment (given in the supplementary masimilar results, which was in line with their results on the local cortical signal 
tests. Importantly, though neither method was able to perfectly recover ground truth signal values, they always mapped them to a desired range of zero to $100 \%$ of ground truth: no negative values were produced, nor values above the original maximum. It is acknowledged that the comparison with $\mathrm{RC}$ on data containing subcortical signal is imperfect, because RC is not designed for this purpose. Nevertheless, the results of the comparison demonstrate why existing projection techniques are unsuitable for data that contains a subcortical signal 430 of interest.

The lsQR methods performed extremely well on some isolated experiments, notably those of the volume to surface to volume design, for which lsQR was used to perform the latter surface to volume projection by inverting the prior volume to surface output. It is likely that good performance for this step was due to the surface to volume projection being over-determined (there are multiple surface vertices per voxel) and hence the matrix inversion is favourable. By contrast, when lsQR was used to perform a volume to surface projection, which is under-determined, higher RMS error and numerical instability was observed (see figures 11 and 13). In particular, lsQR methods returned maximum and minimum signal values outside of the desired range of zero to $100 \%$ : both negative values and values over $100 \%$ of ground truth were observed. These findings show both the promise and pitfalls of working with an inverse-based method: the potential for excellent recovery of ground truth (including the removal of PVE scaling effects), but at the risk of numerical instability leading to signal values far outside of the desired range, which would be a substantial hinderance for any quantitative analysis strategy. It is for this reason that Lonjaret et al. devoted significant effort in their work to regularising the inversion step, but in doing so made the method specific to BOLD [19].

\section{Conclusions}

Toblerone is an extension and generalisation of existing approaches to projection that is able to work with arbitrary data, without making modality-specific 
assumptions, and project to and from hybrid space, a close analogue of the HCP's grayordinates space. The geometric construction of the projection is closely related to the HCP's RC method, and accordingly we observed that the two methods produced similar outputs when operating in a conventional volume-surface sense on data that contains only cortical signal. When operating in hybrid space on data that contains both cortical and subcortical signal, larger and statistically significant differences compared to RC were observed. This implies that the novel aspects of Toblerone's projection are of consequence and make it better suited to working with such data.

\section{Acknowledgements}

The authors would like to thank Tim Coalson of Washington University in St Louis, USA, for advice concerning the correct ordering of triangle vertices. Funding was provided by the EPSRC (EP/P012361/1) and (for T. F. Kirk) the Bellhouse scholarship at Magdalen College, Oxford. HCP data was provided by the Human Connectome Project, WU-Minn Consortium (Principal Investigators: David Van Essen and Kamil Ugurbil; 1U54MH091657) funded by the 16 NIH Institutes and Centers that support the NIH Blueprint for Neuroscience Research; and by the McDonnell Center for Systems Neuroscience at Washington University.

\section{Declarations of interest}

Prior work on the Toblerone software package (related to partial volume estimation) is the subject of a patent application with the US Patent and Trademark Office. The application may be extended to cover aspects of this work. The authors declare no other conflicts of interest.

\section{Data availability}

घ The Toblerone software package is publicly available at https://github. com/tomfrankkirk/toblerone. The analysis code used to perform the ex- 
periments presented in this work may be accessed at https://github.com/

\section{Supplementary material}

Global cortical signal, volume $\rightarrow$ surface $\rightarrow$ volume
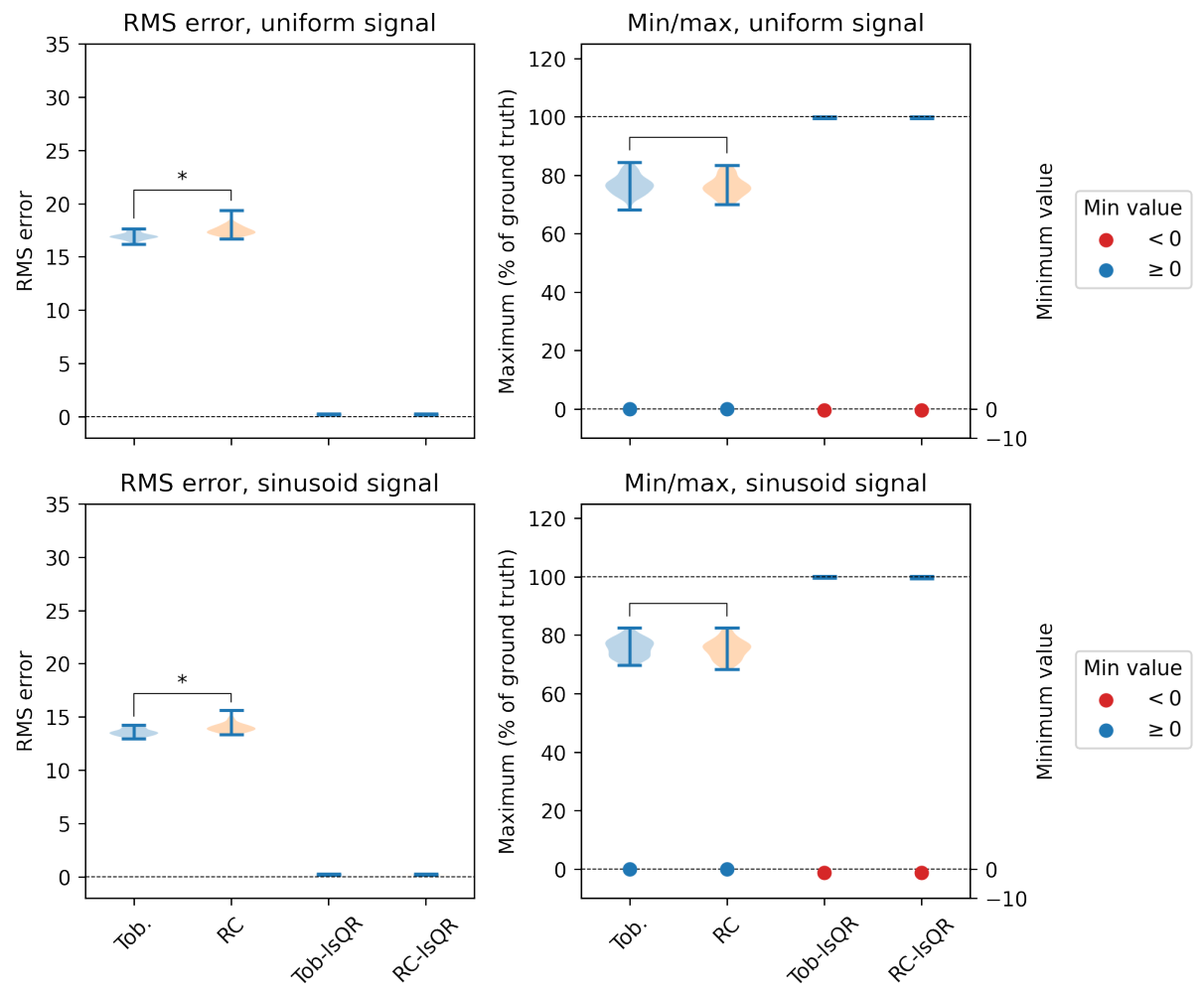

Figure 10: * denotes a comparison of statistical significance. 
Global cortical signal, surface $\rightarrow$ volume $\rightarrow$ surface
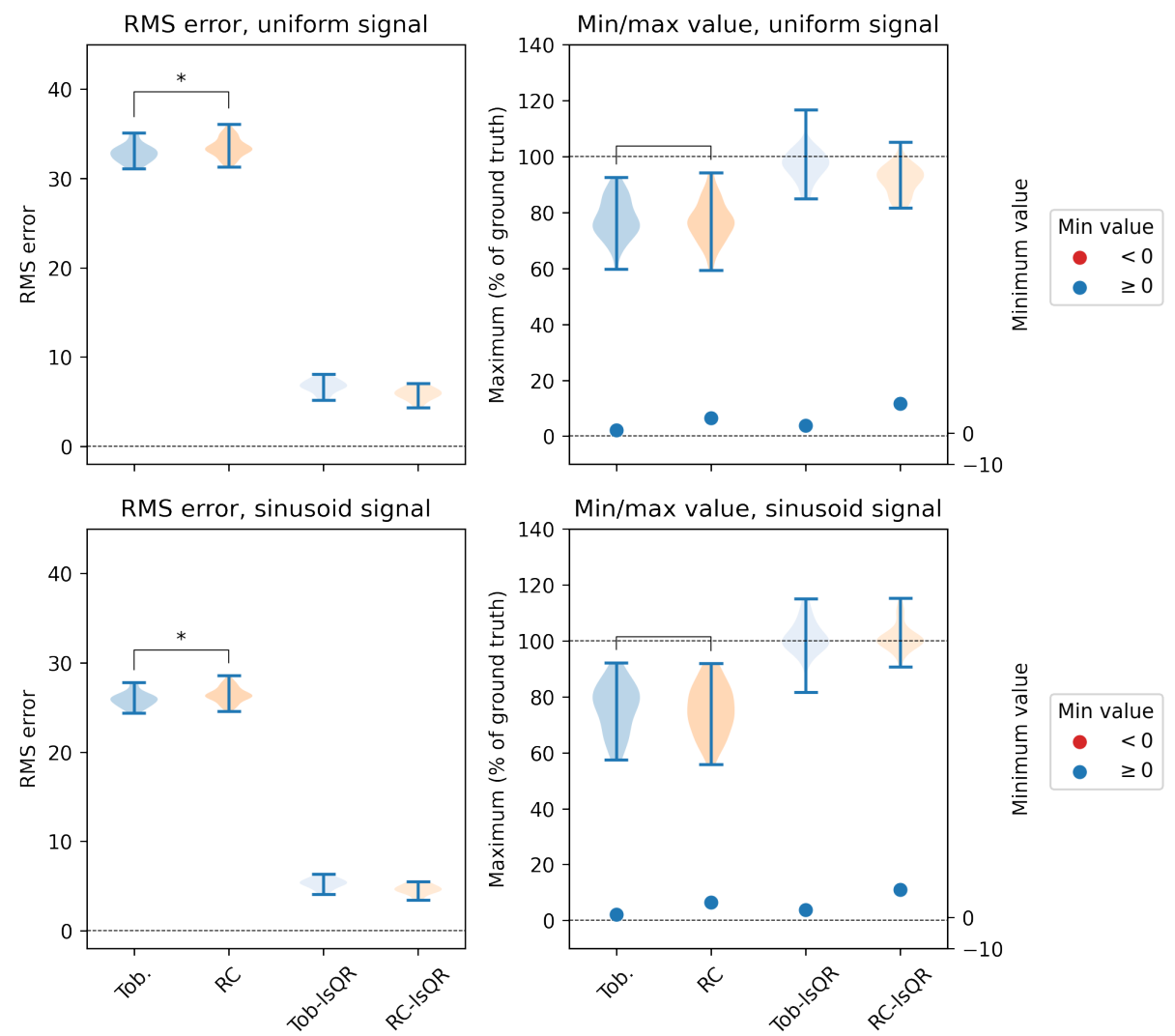

Figure 11: * denotes a comparison of statistical significance. 
Global cortical and subcortical signal, volume $\rightarrow$ surface $\rightarrow$ volume
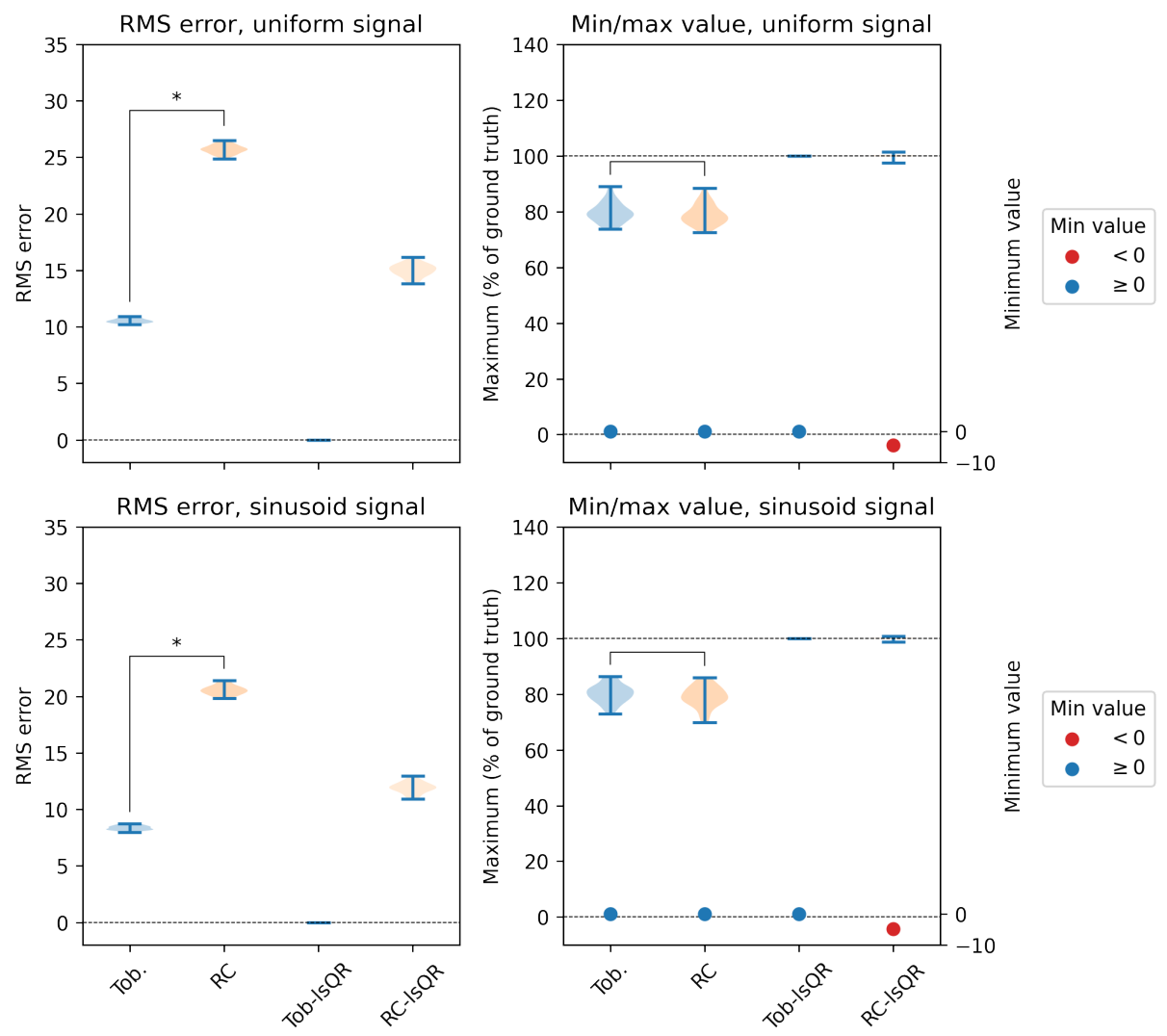

Figure 12: * denotes a comparison of statistical significance. 


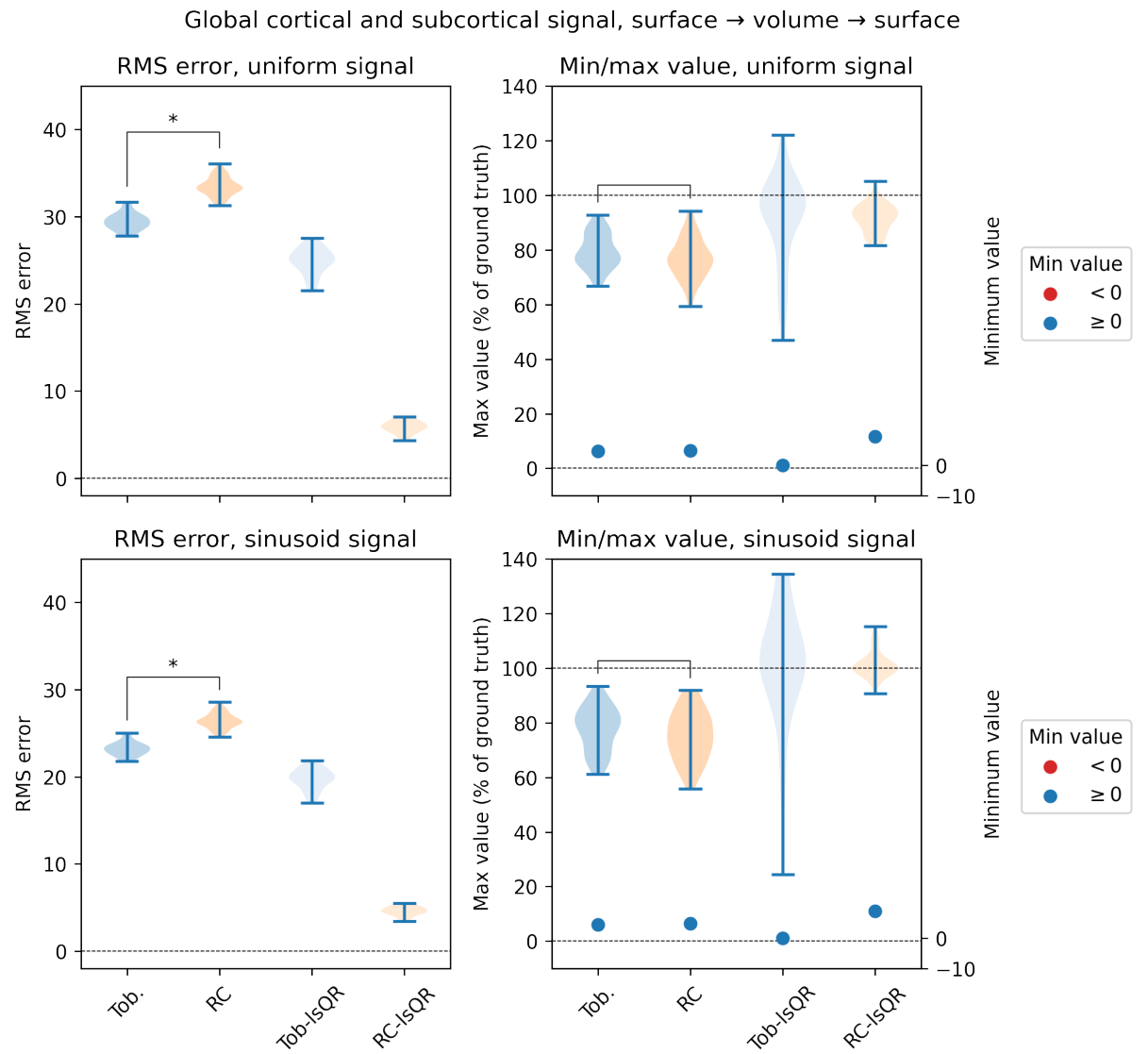

Figure 13: * denotes a comparison of statistical significance.

\section{References}

[1] Dale AM, Fischl B, Sereno MI. Cortical Surface-Based Analysis. NeuroImage 1999;9(2):179-94. URL: http://linkinghub.elsevier. com/retrieve/pii/S1053811998903950 doi:10.1006/nimg.1998.0395 arXiv:nimg.1998.0396.

[2] Frost MA, Goebel R. Measuring structural-functional correspondence: Spatial variability of specialised brain regions after macro-anatomical alignment. NeuroImage 2012;59(2):1369-81. URL: http://dx.doi. org/10.1016/j.neuroimage.2011.08.035 doi $10.1016 / j . n e u r o i m a g e$. 2011.08 .035 
[3] Greve DN, Svarer C, Fisher PM, Feng L, Hansen AE, Baare W, et al. Cor-

tical surface-based analysis reduces bias and variance in kinetic modeling of brain PET data. NeuroImage 2014;92:225-36. URL: http://dx.doi.

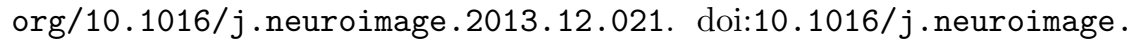
2013.12.021.

[4] Tucholka A, Fritsch V, Poline JB, Thirion B. An empirical comparison of surface-based and volume-based group studies in neuroimaging. NeuroImage 2012;63(3):1443-53. URL: http://dx.doi.org/10.1016/j. neuroimage.2012.06.019, doi:10.1016/j.neuroimage.2012.06.019.

[5] Glasser MF, Smith SM, Marcus DS, Andersson JLR, Auerbach EJ, Behrens TEJ, et al. The Human Connectome Project's neua https://pubmed.ncbi.nlm.nih.gov/27571196https://www.ncbi.nlm. nih.gov/pmc/articles/PMC6172654/, doi/10.1038/nn.4361.

[6] Coalson TS, Van Essen DC, Glasser MF. Lost in Space: The Impact of Traditional Neuroimaging Methods on the Spatial Localization of Cortical Areas. bioRxiv 2018;:255620.

[7] Fischl B, Dale AM. Measuring the thickness of the human cerebral cortex. NeuroImage 1999;9(6 PART II).

[8] Zhao MY, Mezue M, Segerdahl AR, Okell TW, Tracey I, Xiao Y, et al. A systematic study of the sensitivity of partial volume correction methods for the quantification of perfusion from pseudocontinuous arterial spin labeling MRI. NeuroImage 2017;162:38497. URL: https://www.sciencedirect.com/science/article/pii/ S1053811917307103 doi 10.1016/J. NEUROIMAGE.2017.08.072.

[9] Grova C, Makni S, Flandin G, Ciuciu P, Gotman J, Poline JB. Anatomically informed interpolation of fMRI data on the cortical surface. NeuroImage 2006;31(4):1475-86. doi:10.1016/j.neuroimage.2006.02.049 
[10] Operto G, Bulot R, Anton JL, Coulon O. Projection of fMRI data onto the cortical surface using anatomically-informed convo-

1. lution kernels. NeuroImage 2008;39(1):127-35. URL: http://

${ }_{525}$. linkinghub.elsevier.com/retrieve/pii/S1053811907007586. doi 10. $1016 / j$.neuroimage.2007.08.039.

[11] Glasser MF, Sotiropoulos SN, Wilson JA, Coalson TS, Fischl B, Andersson JL, et al. The minimal preprocessing pipelines for the Human Con-

\ nectome Project. NeuroImage 2013;80:105-24. URL: http://dx.doi. 530 org/10.1016/j.neuroimage.2013.04.127 doi 10.1016/j.neuroimage. 2013.04.127, arXiv:NIHMS150003,

[12] Van Essen DC, Ugurbil K, Auerbach E, Barch D, Behrens TE, Bucholz R, et al. The Human Connectome Project: A data acquisition perspective. 2012. doi $10.1016 / \mathrm{j}$.neuroimage.2012.02.018.

${ }_{535}$ [13] Coalson T, Glasser M, Harwell J, Essen DV, Jenkinson M, Oostenveld R. CIFTI-2 Connectivity File Formats Documentation CIFTI working group.

a Tech. Rep.; CIFTI working group; 2014. URL: https://www.nitrc.org/ projects/cifti/.

[14] Fischl B. FreeSurfer. Neuroimage 2012;62(2):774-81. doi:10.1016/j. neuroimage.2012.01.021.FreeSurfer, arXiv:0905267106.

[15] Kirk TF, Coalson TS, Craig MS, Chappell MA. Toblerone: surfacebased partial volume estimation. IEEE Transactions on Medical Imaging 2020;39(5):1501-10. doi:10.1109/TMI .2019.2951080

[16] Dompierre J, Labbe P, Vallet MG, Camarero R. How to Subdivide Pyra${ }_{545}$ mids, Prisms, and Hexahedra into Tetrahedra. In: Proceedings, 8th International Meshing Roundtable. 1999, p. pp.195-204.

[17] Barber CB, Dobkin DP, Huhdanpaa H. The Quickhull Algorithm for 口 Convex Hulls. ACM Trans Math Softw 1996;22(4):469-83. URL: http: //doi.acm.org/10.1145/235815.235821, doi 10.1145/235815.235821. 
[18] Meyer M, Desbrun M, Schröder P, Barr AH. Discrete Differential-Geometry Operators for Triangulated 2-Manifolds BT - Visualization and Mathematics III. Berlin, Heidelberg: Springer Berlin Heidelberg. ISBN 978-3-66205105-4; 2003, p. 35-57.

[19] Lonjaret LT, Bakhous C, Boutelier T, Takerkart S, Coulon O. ISA an inverse surface-based approach for cortical fMRI data projection. In: 14th IEEE International Symposium on Biomedical Imaging, ISBI 2017, Melbourne, Australia, April 18-21, 2017. IEEE. ISBN 978-1-5090-1172-8; 2017, p. 1104-7. URL: https://doi.org/10.1109/ISBI.2017.7950709. doi $10.1109 /$ ISBI.2017.7950709.

[20] Paige CC, Saunders MA. LSQR: An Algorithm for Sparse Linear Equations and Sparse Least Squares. ACM Trans Math Softw 1982;8(1):43-71. URL: https://doi.org/10.1145/355984.355989, doi $10.1145 / 355984$. 355989. 